\title{
Lipid Membranes and Reactions at Lipid Interfaces: Theory, Experiments, and Applications
}

\author{
Ana-Nicoleta Bondar ${ }^{1}$ (D) . Sandro Keller ${ }^{2}$ (D)
}

Received: 3 June 2018 / Accepted: 25 June 2018 / Published online: 29 June 2018

(c) Springer Science+Business Media, LLC, part of Springer Nature 2018

Lipid membranes that surround each biological cell and its various compartments host chemical reactions of paramount importance for the cell. Proteins embedded in the lipid membrane catalyze chemical reactions, transport ions and larger solutes, and participate in cell signaling pathways. Motions of the membrane protein during its function couple to the surrounding lipid membrane, and the physical chemical properties of the membrane can impact protein activity. A detailed description of how membrane proteins work and of the role of lipids in membrane protein function is thus of paramount importance for understanding general physicochemical principles of reactions at the interfaces formed by biological membranes.

This Special Issue presents 18 contributions that discuss state-of-the-art experimental and theoretical studies of lipid membranes and membrane proteins.

The transport of ions and solutes across biological membranes occurs via specialized membrane transporters whose reaction cycles involve protein dynamics and protein conformational changes. For proton-transfer proteins, a fundamental open question is how extended hydrogen-bond networks of the protein participate in proton transfers and the control of protein conformational dynamics.

From atomistic simulations and analyses of water dynamics and hydrogen bond networks in cytochrome $c$ oxidase, Ghane et al. (2018) present a compelling view of the coupling between the protonation state of a key glutamic acid

Ana-Nicoleta Bondar

nbondar@zedat.fu-berlin.de

Sandro Keller

mail@sandrokeller.com

1 Freie Universität Berlin, Department of Physics, Theoretical Molecular Biophysics Group, Arnimallee 14, 14195 Berlin, Germany

2 Molecular Biophysics, Technische Universität Kaiserslautern (TUK), Erwin-Schrödinger-Str. 13, 67663 Kaiserslautern, Germany residue and the conformation of an asparagine thought to function as a gate. Such a tight coupling between protonation state and protein conformational dynamics, which could be a more general feature of protonation-coupled proteins, highlights the usefulness of computer simulation techniques for studying the mechanisms of proton transporters.

The paper by Elghobashi-Meinhardt et al. (2018) presents detailed computations with combined quantum mechanics/ molecular mechanics (QM/MM) demonstrating that hydrogen bonding at the active site of bacteriorhodopsin maintains the retinal chromophore in a twisted geometry. This pre-twist reduces the high energetic cost associated with isomerization of a retinal double bond; thus, recovery of the initial isomeric state of the retinal chromophore can occur with a rate-limiting energy barrier that is compatible with the reaction cycle.

The SecY/Sec61 protein translocon is a protein channel that allows soluble secretory proteins newly synthesized in the cell to be transported to the outer side of the bacterial cell membrane, and transmembrane helices to be inserted into the lipid membrane. The review by Knyazev et al. (2018) presents a comprehensive view of the current status and key open questions regarding the functioning of the protein translocon, including protein conformational dynamics, voltage gating, and the role of the proton motive force (pmf). An intriguing model is proposed for pmf-driven protein translocation, whereby the transmembrane potential helps ensure that movement of a peptide through the translocon is unidirectional.

Computer simulations can provide an atomic-detail view of the structures and associated energetics pertinent to the insertion of hydrophobic peptides into lipid membranes. Gumbart et al. (2018) present an extensive set of simulations demonstrating good agreement for insertion free energies obtained from potential of mean force computations vs. equilibrium simulations, and with two different force fields, which highlights the robustness of the computational approach used. 
Interactions between transmembrane protein helices are involved in, among others, oligomerization of membrane proteins, protein biosynthesis, and cell signaling. The review article by Pawar and Sengupta (2018) highlights the central role of the lipid membrane in the formation of dimers of the transmembrane segments of two ErbB receptors, that is, single-spanning membrane proteins involved in cell signaling. Changes in the lipid membrane composition associated with disease could influence protein/protein interactions.

Evidence for the role of lipids in the functioning of a membrane protein is discussed in the review article by Pashkowsky et al. (2018), who focus on intramembrane proteases: membrane-embedded enzymes that cleave transmembrane substrates. Both the rhomboid proteases and the more complex $\gamma$-secretase are analyzed. An intriguing question is whether the lipid composition of different cellular membranes fine-tunes the functioning of the associated intramembrane protease.

By combining site-directed labeling with steady-state and time-resolved fluorescence spectroscopy, Kyrychenko et al. (2018) first estimated the position in the lipid membrane of the diphtheria toxin translocation domain. This information from experiments was then used for atomistic molecular dynamics simulations, which indicated that membrane partitioning of the protein is energetically more favorable when a particular glutamic acid group is protonated-thus providing a molecular interpretation of the $\mathrm{pH}$ dependence of membrane partitioning.

The orientation of a membrane protein relative to the membrane normal and its location relative to the center of the lipid bilayer are essential to understand, because they could impact molecular interactions and dynamics in the interior of the protein. Simakov and Kurnikova (2018) present continuum electrostatics computations indicating that features of the ionic current for $\alpha$-hemolysin depend on the position of the channel in the membrane, which in turn suggests that this dependence could be used to place the ion channel in the membrane such that electrophysiological properties found from computations are compatible with experimental results.

Electrophysiological measurements and an analytical model for the physical interpretation of ionic currents through the $\alpha$-hemolysin nanopore are reported by Asandei et al. (2018), who document an experimental setting in which a poly-amidoamine dendrimer, with a diameter of $\sim 25 \AA$, is thought to be stretched such that it can pass through the nanopore of $\alpha$-hemolysin (estimated diameter of $\sim 15 \AA$ ) at low $\mathrm{pH}$, where carboxylate groups from the outer region of the dendrimer might protonate.

A molecular picture of the three-dimensional structure of an ion channel is essential for deciphering its mechanism of action. Andersson et al. (2018) rely on homology modeling and molecular simulations to derive a structural model of the viral potassium channel $\mathrm{Kcv}_{\mathrm{PBCV}-1}$; they further validate the model by assessing interactions with five compounds known to block the ion channel and by studying ion conductance. Dynamics at the selectivity filter is suggested to contribute to fast gating of the ion channel.

Dynamics of lipid membranes involves flip-flop of lipid molecules, a process whereby lipid molecules exchange from one membrane leaflet to the other. UV/VIS and fluorescence experiments presented by Coreta-Gomes et al. (2018) indicate that translocation between leaflets becomes faster than desorption from the lipid bilayer when the number of carbon atoms in the alkyl chain is at least 14. Experimental observations provide the basis for discussing implications for protocols used to prepare asymmetric liposomes or to ensure the homogeneity of liposomes.

In a nanodisc, the dynamics of lipids is constrained by the confines of the molecules used to assemble the nanodisc. The significant interest in using nanodiscs for probing the functioning of membrane proteins highlights the importance of characterizing the physicochemical properties of such nanodiscs and molecules that constitute them. Grethen et al. (2018) present time-resolved Förster resonance energy transfer experiments on lipid nanodiscs encapsulated by styrene/maleic acid, SMA (2:1). Their experiments indicate that transfer of lipids between these nanodiscs, which is faster than in protein-encapsulated nanodiscs and is promoted by increased ionic strength, occurs via a mechanism that depends on collisional lipid exchange at millimolar lipid concentrations, as compared with diffusional transfer at lower concentrations of lipids.

Biological lipid membranes present to the aqueous bulk a highly polar interface contributed by the lipid headgroups: phosphatidylcholine (PC) and phosphatidylethanolamine (PE) lipids, which are major constituents of eukaryotic cell membranes, are zwitterionic; another major lipid, phosphatidylserine, is negatively charged. Over the years, significant research efforts have been dedicated to the understanding of how water molecules, ions, and biomolecules interact with lipid membrane interfaces. The review by Friedman (2018) discusses experimental and theoretical works on the binding of ions to lipid membranes, including the understudied membranes of archaea. Physicochemical properties of the ions and features of the molecular dynamics approach provide the bases for discussing implications for simulations of membrane/ions interactions.

Hydrogen-bonded water networks at lipid membrane interfaces could participate in the binding and lateral transfer of protons. Karathanou and Bondar (2018) present an algorithm for identifying water/phosphate hydrogen-bond networks at the lipid headgroup interface, and for characterizing the dynamics of hydrogen-bonded lipid phosphate clusters. Linear clusters of lipid phosphates bridged by a hydrogen-bonded water molecule can extend to transiently 
bridge up to six lipid molecules. Overall, water-mediated clusters of lipid headgroups are highly dynamic.

Tremi et al. (2018) combine experiments and numerical simulations to characterize model lipid membranes that contain TSAP - a PC with a mono-trans isomer of arachidonic acid moiety that might be relevant for human health and disease. Simulations, together with the dynamic light scattering and atomic force microscopy experiments, suggest that the presence of TSAP associates with decreased area per lipid and increased ordering of the membrane.

Cholesterol is an essential ingredient of the plasma membrane of eukaryotes, and its influence on the structure and dynamics of lipid membrane has been studied extensively with experiments and computations. In this Special Issue, three contributions bring new insights into how the presence of cholesterol alters physicochemical properties of lipid membranes:

Rezelj et al. (2018) report a protocol to prepare lipid vesicles and nanodiscs containing archaeal lipids and cholesterol, and extensive biophysical studies to characterize the vesicles and the binding to these vesicles of two different cytolysins-toxins whose pore-forming properties depend on the presence of cholesterol. The high stability of the vesicles made of archaeal lipids is suggested to render them attractive for biotechnology applications and for studies of membrane/protein interactions.

Oakes and Domene (2018) use atomistic molecular dynamics simulations to study POPC with three different isomers of cholesterol-native cholesterol, its enantiomer, and its epi-stereoisomer-to dissect the impact of cholesterol on the membrane dipole moment. Unlike the cases of native cholesterol and its enantiomer, the solvent-accessible surface area remains close to that of pure POPC in the presence of epi-cholesterol.

The computations by Owen et al. (2018) document the protective effect that cholesterol can have on oxidized POPC membranes. Simulations were performed for POPC/cholesterol membranes with various concentrations of two types of oxidized lipids, which have an aldehyde vs. carboxylic acid modification in their sn-2 alkyl chain. Hydrogen bonds between cholesterol and oxidized lipids are found to help maintain the structural integrity of the lipid membrane.

Knowledge of how chemical reactions occur at membrane interfaces is essential for understanding fundamental biological phenomena and as a basis for new biomedical applications, and combining experimental with theoretical approaches has the potential to provide detailed understanding of events that occur at complex interfaces formed by biological lipid membranes. It is in this spirit that the Special Issue on Lipid Membranes and Reactions at Membrane Interfaces brings together articles highlighting exciting developments in theoretical and experimental membrane biophysics.
Acknowledgements We thank the Authors for making this Special Issue possible. A-NB acknowledges funding from the Freie Universität Berlin within the Excellence Initiative of the German Research Foundation. SK acknowledges funding from the Carl Zeiss Foundation through the Centre for Lipidomics (CZSLip) and the Deutsche Forschungsgemeinschaft (DFG) through International Research Training Group (IRTG) 1830 .

\section{References}

Andersson AEV, Kasimova MA, Delemotte L (2018) Exploring the viral channel $\mathrm{Kcv}_{\mathrm{PBCV}-1}$ function via computation. J Membr Biol. https://doi.org/10.1007/s00232-018-0022-2

Asandei A, Schiopu I, Ciobanasu C, Park Y, Luchian T (2018) If squeezed, a camel passes through the eye of a needle: voltagemediated stretching of dendrimers facilitates passage through a nanopore. J Membr Biol. https://doi.org/10.1007/s0023 2-017-9999-1

Coreta-Gomes FM, Vaz WLC, Moreno MJ (2018) Effect of acyl chain length on the rate of phospholipid flip-flop and intermembrane transfer. J Membr Biol. https://doi.org/10.1007/s0023 2-017-0009-4

Elghobashi-Meinhardt N, Phatak P, Bondar A-N, Elstner M, Smith JC (2018) Catalysis of ground state cis $\rightarrow$ trans isomerization of bacteriorhodopsin's retinal chromophore by a hydrogen-bond network. J Membr Biol. https://doi.org/10.1007/s00232-018-0027-x

Friedman R (2018) Membrane-ion interactions. J Membr Biol. https ://doi.org/10.1007/s00232-017-0010-y

Ghane T, Gorriz RF, Wrzalek S, Volkenandt S, Dalatieh F, Reidelbach M, Imhof P (2018) Hydrogen-bonded network and water dynamics in the D-channel of cytochrome $c$ oxidase. J Membr Biol. https ://doi.org/10.1007/s00232-018-0019-x

Grethen A, Glueck D, Keller S (2018) Role of Coulombic repulsion in collisional lipid transfer among SMA(2:1)-bounded nanodiscs. J Membr Biol. https://doi.org/10.1007/s00232-018-0024-0

Gumbart JC, Ulmschneider MB, Hazel A, White SH, Ulmschneider JP (2018) Computed free energies of peptide insertion into bilayers are independent of computational method. J Membr Biol. https:// doi.org/10.1007/s00232-018-0026-y

Karathanou K, Bondar A-N (2018) Dynamic water hydrogen-bond networks at the interface of a lipid membrane containing palmitoyl-oleoyl phosphatidylglycerol. J Membr Biol. https://doi. org/10.1007/s00232-018-0023-1

Knyazev DG, Kuttner R, Zimmermann M, Sobakinskaya E, Pohl P (2018) Driving forces of translocation through bacterial translocon SecYEG. J Membr Biol. https://doi.org/10.1007/s0023 2-017-0012-9

Kyrychenko A, Lim NM, Vasquez-Montes V, Rodnin MV, Freites JA, Nguyen LP, Tobias DJ, Mobley DL, Ladokhin AS (2018) Refining protein penetration into the lipid bilayer using fluorescence quenching and molecular dynamics simulations: the case of diphtheria toxin translocation domain. J Membr Biol. https:// doi.org/10.1007/s00232-018-0030-2

Oakes V, Domene C (2018) Stereospecific interactions of cholesterol in a model cell membrane: implications for the membrane dipole potential. J Membr Biol. https://doi.org/10.1007/s0023 2-018-0016-0

Owen MC, Kulig W, TRog T, Vattulainen I, Strodel B (2018) Cholesterol protects the oxidized lipid bilayer from water injury: an all-atom molecular dynamics study. J Membr Biol. https://doi. org/10.1007/s00232-018-0028-9

Paschkowsky S, Oestereich F, Munter LM (2018) Embedded in the membrane: how lipids confer activity and specificity to 
intramembrane proteases. J Membr Biol. https://doi.org/10.1007/ s00232-017-0008-5

Pawar AB, Sengupta D (2018) Effect of membrane composition on receptor association: implications of cancer lipidomics on ErbB receptors. J Membr Biol. https://doi.org/10.1007/s0023 2-018-0015-1

Rezelj S, Kozorog M, Švigelj T, Poklar Ulrih N, Žnidaršič N, Podobnik M, Anderluh G (2018) Cholesterol enriched archaeosomes as a molecular system for studying interactions of cholesteroldependent cytolysins with membrane. J Membr Biol. https://doi. org/10.1007/s00232-018-0018-y
Simakov NA, Kurnikova MG (2018) Membrane position dependency of the $\mathrm{p} K_{\mathrm{a}}$ and conductivity of the protein ion channel. J Membr Biol. https://doi.org/10.1007/s00232-018-0013-3

Tremi I, Anagnostopoulos D, Spyratou E, Gkeka P, Georgakilas AG, Chatgilialoglu C, Cournia Z (2018) Effect of 5-trans isomer of arachidonic acid on model liposomal membranes studied by a combined simulation and experimental approach. J Membr Biol. https://doi.org/10.1007/s00232-018-0029-8 